Table S4. List of genes in upper $1 \% \mathrm{~F}_{\mathrm{ST}}$ distribution in comparision of brown layers and white layers.

\begin{tabular}{|c|c|c|c|c|}
\hline Chr & Start & End & Description & Fst \\
\hline 1 & 9325096 & 9459193 & semaphorin-3E precursor & 0.686 \\
\hline 1 & 9561909 & 9881531 & piccolo presynaptic cytomatrix protein & 0.686 \\
\hline 1 & 14213411 & 14241547 & phosphatidylinositol-4,5-bisphosphate 3-kinase catalytic subunit gamma isoform & 0.713 \\
\hline 1 & 14328390 & 14346800 & protein kinase, cAMP-dependent, regulatory, type II, beta & 0.713 \\
\hline 1 & 14356759 & 14367631 & HMG box-containing protein 1 & 0.713 \\
\hline 1 & 14369255 & 14553213 & conserved oligomeric Golgi complex subunit 5 & 0.713 \\
\hline 1 & 14553188 & 14563663 & dihydrouridine synthase 4-like (S. cerevisiae) & 0.713 \\
\hline 1 & 14567866 & 14591310 & B-cell receptor-associated protein 29 & 0.713 \\
\hline 1 & 14621478 & 14632510 & E3 ubiquitin-protein ligase Hakai & 0.713 \\
\hline 1 & 14632208 & 14642808 & chloride anion exchanger & 0.713 \\
\hline 1 & 14666134 & 14679885 & dihydrolipoyl dehydrogenase, mitochondrial & 0.713 \\
\hline 1 & 14681577 & 14715087 & Laminin subunit beta-1 & 0.713 \\
\hline 1 & 14777556 & 14800582 & endoplasmic reticulum-Golgi intermediate compartment protein 2 & 0.713 \\
\hline 1 & 14916517 & 15054085 & solute carrier family 2 (facilitated glucose transporter), member 13 & 0.713 \\
\hline 1 & 24794262 & 24882798 & hepatocyte growth factor receptor precursor & 0.507 \\
\hline 1 & 25847642 & 26191414 & FoxP2; Uncharacterized protein & 0.753 \\
\hline 1 & 26299344 & 26325360 & protein phosphatase 1 , regulatory subunit $3 \mathrm{~A}$ & 0.753 \\
\hline 1 & 28473454 & 28508702 & calcium-independent phospholipase A2-gamma & 0.568 \\
\hline 1 & 31999958 & 32082280 & monocarboxylate transporter 2 & 0.526 \\
\hline 1 & 35865662 & 36009723 & protein tyrosine phosphatase, receptor type, $R$ & 0.607 \\
\hline 1 & 40131212 & 40428161 & protein tyrosine phosphatase, receptor type, f polypeptide (PTPRF), interacting protein (liprin), alpha 2 & 0.560 \\
\hline 1 & 40619495 & 40678658 & methyltransferase like 25 & 0.560 \\
\hline 1 & 42095834 & 42213439 & Alpha-1,3-mannosyl-glycoprotein 4-beta-N-acetylglucosaminyltransferase C & 0.636 \\
\hline 1 & 43171637 & 43175978 & dual specificity protein phosphatase 6 & 0.642 \\
\hline 1 & 43207669 & 43254712 & WD repeat-containing protein 51B & 0.642 \\
\hline 1 & 43273706 & 43305815 & plasma membrane calcium-transporting ATPase 1 & 0.642 \\
\hline 1 & 46488737 & 46510905 & lamina-associated polypeptide 2 , isoform beta & 0.568 \\
\hline
\end{tabular}


potassium channel tetramerization domain containing 17

5135516651362344 thiosulfate sulfurtransferase

5159855051666512 myosin-9

$151759800 \quad 51835231$

\section{RNA binding}

DNA replication licensing factor MCM5

heme oxygenase 1

$152524419 \quad 52799322$

Target of Myb protein 1

0.634

159

$59065375 \quad 59100295$

16043889060496703

UC338

glycosyltransferase-like protein LARGE1

0.634

6342948563435977 dynamin-1-like protein

beta-1,4-N-acetyl-galactosaminyl transferase 3 microsomal glutathione S-transferase 1 tubulin tyrosine ligase-like family, member 1 


\begin{tabular}{|c|c|c|c|c|}
\hline 1 & 70002408 & 70079769 & rho GTPase-activating protein 8 & 0.551 \\
\hline 1 & 70098157 & 70154426 & PHD finger protein 21B & 0.551 \\
\hline 1 & 72902806 & 73148067 & fatty acyl CoA reductase 2 & 0.737 \\
\hline 1 & 84610640 & 84660808 & interphotoreceptor matrix proteoglycan 2 precursor & 0.640 \\
\hline 1 & 84665305 & 84703930 & SUMO1/sentrin specific peptidase 7 & 0.640 \\
\hline 1 & 84714811 & 84724364 & PEST proteolytic signal-containing nuclear protein & 0.640 \\
\hline 1 & 84747544 & 84751604 & ribosomal protein L24 & 0.640 \\
\hline 1 & 84799655 & 84816004 & NF-kappa-B inhibitor zeta & 0.640 \\
\hline 1 & 87878151 & 87933199 & pleckstrin homology-like domain, family B, member 2 & 0.569 \\
\hline 1 & 91358735 & 91424437 & prostaglandin F2 receptor inhibitor & 0.551 \\
\hline 1 & $1 \mathrm{E}+08$ & $1 \mathrm{E}+08$ & neural cell adhesion molecule 2 & 0.587 \\
\hline 1 & $1.08 \mathrm{E}+08$ & $1.08 \mathrm{E}+08$ & Down syndrome cell adhesion molecule & 0.599 \\
\hline 1 & $1.14 \mathrm{E}+08$ & $1.15 \mathrm{E}+08$ & dystrophin & 0.599 \\
\hline 1 & 1.17E+08 & 1.17E+08 & DNA polymerase & 0.672 \\
\hline 1 & $1.17 \mathrm{E}+08$ & $1.18 \mathrm{E}+08$ & phosphate cytidylyltransferase 1 , choline, beta & 0.672 \\
\hline 1 & 1.17E+08 & 1.17E+08 & Small Cajal body specific RNA 24 & 0.672 \\
\hline 1 & $1.19 \mathrm{E}+08$ & $1.2 \mathrm{E}+08$ & SH3 domain-containing kinase-binding protein 1 & 0.510 \\
\hline 1 & $1.24 \mathrm{E}+08$ & $1.24 \mathrm{E}+08$ & chloride channel, voltage-sensitive 4 & 0.575 \\
\hline 1 & $1.45 \mathrm{E}+08$ & $1.45 \mathrm{E}+08$ & UDP-glucose:glycoprotein glucosyltransferase 2 precursor & 0.514 \\
\hline 1 & $1.47 \mathrm{E}+08$ & $1.47 \mathrm{E}+08$ & glypican 5 & 0.656 \\
\hline 1 & $1.49 \mathrm{E}+08$ & $1.49 \mathrm{E}+08$ & SLIT and NTRK-like family, member 5 & 0.821 \\
\hline 1 & $1.52 \mathrm{E}+08$ & $1.52 \mathrm{E}+08$ & NEDD4 family-interacting protein 2 & 0.931 \\
\hline 1 & $1.52 \mathrm{E}+08$ & $1.52 \mathrm{E}+08$ & RNA binding motif protein 26 & 0.931 \\
\hline 1 & $1.52 \mathrm{E}+08$ & $1.52 \mathrm{E}+08$ & protein sprouty homolog 2 & 0.931 \\
\hline 1 & $1.54 \mathrm{E}+08$ & $1.54 \mathrm{E}+08$ & ubiquitin carboxyl-terminal hydrolase isozyme L3 & 0.539 \\
\hline 1 & $1.54 \mathrm{E}+08$ & $1.54 \mathrm{E}+08$ & TBC1 domain family, member 4 & 0.539 \\
\hline 1 & $1.55 \mathrm{E}+08$ & $1.55 \mathrm{E}+08$ & Kruppel-like factor 12 & 0.539 \\
\hline 1 & $1.62 \mathrm{E}+08$ & $1.62 \mathrm{E}+08$ & diaphanous-related formin 3 & 0.623 \\
\hline 1 & $1.64 \mathrm{E}+08$ & $1.64 \mathrm{E}+08$ & Small nucleolar RNA U2-30 & 0.633 \\
\hline 1 & $1.64 \mathrm{E}+08$ & $1.64 \mathrm{E}+08$ & Small nucleolar RNA U2-19 & 0.633 \\
\hline
\end{tabular}




\begin{tabular}{|c|c|c|c|c|}
\hline 1 & $1.65 E+08$ & $1.65 E+08$ & von Willebrand factor A domain containing 8 & 0.683 \\
\hline 1 & $1.66 \mathrm{E}+08$ & $1.66 \mathrm{E}+08$ & diacylglycerol kinase, eta & 0.683 \\
\hline 1 & $1.67 \mathrm{E}+08$ & $1.67 \mathrm{E}+08$ & family with sequence similarity 194 , member B & 0.600 \\
\hline 1 & $1.67 \mathrm{E}+08$ & $1.67 \mathrm{E}+08$ & Spermatid-associated protein & 0.600 \\
\hline 1 & $1.67 \mathrm{E}+08$ & $1.67 \mathrm{E}+08$ & siah E3 ubiquitin protein ligase family member 3 & 0.600 \\
\hline 1 & $1.67 \mathrm{E}+08$ & $1.67 E+08$ & plastin-2 & 0.600 \\
\hline 1 & $1.67 \mathrm{E}+08$ & $1.67 \mathrm{E}+08$ & leucine rich repeat containing 63 & 0.600 \\
\hline 1 & $1.67 \mathrm{E}+08$ & $1.67 \mathrm{E}+08$ & KIAA0226-like & 0.600 \\
\hline 1 & $1.68 \mathrm{E}+08$ & $1.68 \mathrm{E}+08$ & esterase D & 0.600 \\
\hline 1 & $1.68 \mathrm{E}+08$ & $1.68 \mathrm{E}+08$ & 5-hydroxytryptamine (serotonin) receptor $2 \mathrm{~A}, \mathrm{G}$ protein-coupled & 0.600 \\
\hline 1 & $1.68 \mathrm{E}+08$ & $1.68 \mathrm{E}+08$ & succinate-CoA ligase, ADP-forming, beta subunit & 0.600 \\
\hline 1 & $1.68 \mathrm{E}+08$ & $1.68 \mathrm{E}+08$ & integral membrane protein $2 \mathrm{~B}$ & 0.600 \\
\hline 1 & $1.68 \mathrm{E}+08$ & $1.68 \mathrm{E}+08$ & retinoblastoma-associated protein & 0.600 \\
\hline 1 & $1.68 \mathrm{E}+08$ & $1.68 \mathrm{E}+08$ & regulator of chromosome condensation (RCC1) and BTB (POZ) domain containing protein 2 & 0.600 \\
\hline 1 & $1.68 \mathrm{E}+08$ & $1.68 \mathrm{E}+08$ & fibronectin type-III domain-containing protein 3a & 0.600 \\
\hline 1 & $1.68 \mathrm{E}+08$ & $1.68 \mathrm{E}+08$ & motilin receptor & 0.600 \\
\hline 1 & $1.68 \mathrm{E}+08$ & $1.69 \mathrm{E}+08$ & calcium binding protein 39 -like & 0.600 \\
\hline 1 & $1.75 \mathrm{E}+08$ & $1.75 \mathrm{E}+08$ & microtubule associated tumor suppressor candidate 2 & 0.549 \\
\hline 1 & $1.76 \mathrm{E}+08$ & $1.76 \mathrm{E}+08$ & cyclin-dependent kinase 8 & 0.566 \\
\hline 1 & $1.76 \mathrm{E}+08$ & $1.76 \mathrm{E}+08$ & ring finger protein ( $\mathrm{C} 3 \mathrm{H} 2 \mathrm{C} 3$ type) 6 & 0.566 \\
\hline 1 & $1.76 \mathrm{E}+08$ & $1.76 \mathrm{E}+08$ & ATPase, aminophospholipid transporter, class I, type $8 \mathrm{~A}$, member 2 & 0.566 \\
\hline 1 & $1.77 \mathrm{E}+08$ & $1.77 \mathrm{E}+08$ & spastic ataxia of Charlevoix-Saguenay (sacsin) & 0.581 \\
\hline 1 & $1.78 \mathrm{E}+08$ & $1.78 \mathrm{E}+08$ & glia-activating factor & 0.581 \\
\hline 1 & $1.85 \mathrm{E}+08$ & $1.85 \mathrm{E}+08$ & solute carrier family 36 (proton/amino acid symporter), member 4 & 0.741 \\
\hline 1 & $1.91 \mathrm{E}+08$ & $1.92 \mathrm{E}+08$ & teneurin transmembrane protein 4 & 0.711 \\
\hline 1 & $1.92 E+08$ & $1.92 \mathrm{E}+08$ & asparaginyl-tRNA synthetase 2 , mitochondrial (putative) & 0.711 \\
\hline 1 & $1.93 \mathrm{E}+08$ & $1.93 \mathrm{E}+08$ & tsukushin precursor & 0.593 \\
\hline 2 & 5408261 & 5613834 & sodium channel, voltage-gated, type $V$, alpha subunit & 0.561 \\
\hline 2 & 13816140 & 13922505 & neuropilin-1 precursor & 0.600 \\
\hline 2 & 13975831 & 14017232 & integrin beta-1 precursor & 0.600 \\
\hline
\end{tabular}




\begin{tabular}{|c|c|c|c|c|}
\hline 2 & 13771061 & 13771230 & TUC338 & 0.600 \\
\hline 2 & 19881822 & 19979217 & ras suppressor protein 1 & 0.581 \\
\hline 2 & 24256193 & 24345252 & calcium-binding mitochondrial carrier protein Aralar2 & 0.525 \\
\hline 2 & 24450646 & 24457181 & split hand/foot malformation (ectrodactyly) type 1 & 0.525 \\
\hline 2 & 45255770 & 45389575 & cytoplasmic linker associated protein 2 & 0.535 \\
\hline 2 & 45413332 & 45443733 & programmed cell death 6 -interacting protein & 0.535 \\
\hline 2 & 50484818 & 50543299 & cyclin-dependent kinase 13 & 0.751 \\
\hline 2 & 50564486 & 50571264 & M-phase specific PLK1 interacting protein & 0.751 \\
\hline 2 & 57910351 & 57932198 & glycosylphosphatidylinositol specific phospholipase D1 & 0.632 \\
\hline 2 & 62603034 & 62615535 & phosphatase and actin regulator 1 & 0.531 \\
\hline 2 & 67611323 & 67673626 & myosin light chain kinase family, member 4 & 0.542 \\
\hline 2 & 67719974 & 67729598 & serpin B6 & 0.542 \\
\hline 2 & 67730365 & 67739883 & Heterochromatin-associated protein MENT & 0.542 \\
\hline 2 & 67745615 & 67751998 & Heterochromatin-associated protein MENT & 0.542 \\
\hline 2 & 67753130 & 67763712 & serpin peptidase inhibitor, clade B (ovalbumin), member 2 & 0.542 \\
\hline 2 & 67771785 & 67779357 & Ovalbumin & 0.542 \\
\hline 2 & 67790408 & 67796318 & ovalbumin-related protein $Y$ & 0.542 \\
\hline 2 & 74276764 & 74466274 & Cadherin; Uncharacterized protein & 0.632 \\
\hline 2 & 76311201 & 76496830 & trio Rho guanine nucleotide exchange factor & 0.518 \\
\hline 2 & 78393973 & 78403910 & T-complex protein 1 subunit epsilon & 0.614 \\
\hline 2 & 78404319 & 78411897 & family with sequence similarity 173, member B & 0.614 \\
\hline 2 & 79220949 & 79424359 & adenylate cyclase 2 (brain) & 0.541 \\
\hline 2 & 80447666 & 80505627 & von Willebrand factor $\mathrm{C}$ domain containing 2 & 0.633 \\
\hline 2 & 80510980 & 80537096 & Zona pellucida-binding protein 1 & 0.633 \\
\hline 2 & 80612647 & 80685641 & DNA-binding protein Ikaros & 0.633 \\
\hline 2 & 80708843 & 80754977 & dopa decarboxylase (aromatic L-amino acid decarboxylase) & 0.633 \\
\hline 2 & 83099424 & 83462516 & formin homology 2 domain containing 3 & 0.566 \\
\hline 2 & 84812789 & 85025689 & molybdenum cofactor sulfurase & 0.699 \\
\hline 2 & 86065381 & 86068294 & iroquois homeobox 4 & 0.766 \\
\hline 2 & 97144112 & 97156128 & cell death activator CIDE-A & 0.550 \\
\hline
\end{tabular}




\begin{tabular}{|c|c|c|c|c|}
\hline 2 & $1.03 E+08$ & $1.03 E+08$ & RIO kinase 3 & 0.559 \\
\hline 2 & $1.03 E+08$ & $1.03 E+08$ & Niemann-Pick disease, type $\mathrm{C} 1$ & 0.559 \\
\hline 2 & $1.03 E+08$ & $1.03 E+08$ & ankyrin repeat domain 29 & 0.559 \\
\hline 2 & $1.03 E+08$ & $1.03 E+08$ & laminin, alpha 3 & 0.559 \\
\hline 2 & $1.03 \mathrm{E}+08$ & $1.03 E+08$ & Oxysterol-binding protein & 0.559 \\
\hline 2 & $1.08 \mathrm{E}+08$ & $1.08 \mathrm{E}+08$ & U6 spliceosomal RNA & 0.553 \\
\hline 2 & $1.1 \mathrm{E}+08$ & $1.1 \mathrm{E}+08$ & V-type proton ATPase subunit $\mathrm{H}$ & 0.614 \\
\hline 2 & $1.1 \mathrm{E}+08$ & $1.1 \mathrm{E}+08$ & Regulator of G-protein signaling 20 & 0.614 \\
\hline 2 & $1.1 \mathrm{E}+08$ & $1.1 \mathrm{E}+08$ & transcription elongation factor $A$ protein 1 & 0.614 \\
\hline 2 & $1.11 \mathrm{E}+08$ & $1.11 \mathrm{E}+08$ & tyrosine-protein kinase Lyn & 0.529 \\
\hline 2 & 1.17E+08 & $1.17 E+08$ & telomeric repeat-binding factor 1 & 0.523 \\
\hline 2 & $1.23 \mathrm{E}+08$ & $1.23 E+08$ & cyclic nucleotide gated channel beta 3 & 0.611 \\
\hline 2 & $1.24 \mathrm{E}+08$ & $1.24 \mathrm{E}+08$ & nibrin & 0.532 \\
\hline 2 & $1.24 \mathrm{E}+08$ & $1.24 E+08$ & Calbindin & 0.532 \\
\hline 2 & $1.27 \mathrm{E}+08$ & $1.27 \mathrm{E}+08$ & Iysosomal protein transmembrane 4 beta & 0.523 \\
\hline 2 & $1.28 \mathrm{E}+08$ & $1.28 \mathrm{E}+08$ & vacuolar protein sorting 13 homolog B (yeast) & 0.537 \\
\hline 2 & $1.28 \mathrm{E}+08$ & $1.28 \mathrm{E}+08$ & cytochrome $\mathrm{c}$ oxidase subunit $6 \mathrm{C}$ & 0.537 \\
\hline 2 & $1.37 \mathrm{E}+08$ & $1.37 \mathrm{E}+08$ & hyaluronan synthase 2 & 0.579 \\
\hline 2 & 1.47E+08 & $1.47 \mathrm{E}+08$ & t-SNARE domain containing 1 & 0.530 \\
\hline 3 & 8094160 & 8384451 & anaplastic lymphoma receptor tyrosine kinase & 0.519 \\
\hline 3 & 9521292 & 9539167 & protein pellino homolog 1 & 0.560 \\
\hline 3 & 13302980 & 13394073 & 1-phosphatidylinositol-4,5-bisphosphate phosphodiesterase beta-4 & 0.628 \\
\hline 3 & 20511715 & 20538253 & N-lysine methyltransferase SMYD2 & 0.580 \\
\hline 3 & 28093815 & 28285437 & dishevelled associated activator of morphogenesis 2 & 0.716 \\
\hline 3 & 28650945 & 28730284 & glucagon-like peptide 1 receptor precursor & 0.716 \\
\hline 3 & 28737986 & 28830043 & dynein, axonemal, heavy chain 8 & 0.716 \\
\hline 3 & 28867771 & 28979903 & BTB/POZ domain-containing protein 9 & 0.716 \\
\hline 3 & 29337956 & 29424515 & MAM domain-containing glycosylphosphatidylinositol anchor protein 1 precursor & 0.716 \\
\hline 3 & 29519203 & 29541046 & FtsJ methyltransferase domain containing 2 & 0.716 \\
\hline 3 & 29557966 & 29574363 & probable alanyl-tRNA synthetase, mitochondrial & 0.716 \\
\hline
\end{tabular}




\begin{tabular}{|c|c|c|c|c|}
\hline 3 & 29217044 & 29217179 & TUC338 & 0.716 \\
\hline 3 & 40939403 & 41033678 & SPARC related modular calcium binding 2 & 0.671 \\
\hline 3 & 41121124 & 41158394 & sulfotransferase & 0.671 \\
\hline 3 & 41160297 & 41172875 & dishevelled-binding antagonist of beta-catenin 2 & 0.671 \\
\hline 3 & 41393868 & 41474285 & myeloid/lymphoid or mixed-lineage leukemia (trithorax homolog, Drosophila); translocated to, 4 & 0.671 \\
\hline 3 & 41687479 & 41708127 & unc-93 homolog A (C. elegans) & 0.671 \\
\hline 3 & 41711697 & 41713292 & tubulin tyrosine ligase-like family, member 2 & 0.671 \\
\hline 3 & 41759132 & 41766064 & C-C chemokine receptor type 6 & 0.671 \\
\hline 3 & 41783071 & 41803951 & FGFR1 oncogene partner & 0.671 \\
\hline 3 & 41810099 & 41831299 & ribonuclease T2 precursor & 0.671 \\
\hline 3 & 41958446 & 42114412 & ribosomal protein S6 kinase, 90kDa, polypeptide 2 & 0.671 \\
\hline 3 & 42131326 & 42141404 & brain protein 44 -like protein & 0.671 \\
\hline 3 & 42233586 & 42241528 & brachyury protein & 0.671 \\
\hline 3 & 42463951 & 42626252 & phosphodiesterase $10 \mathrm{~A}$ & 0.671 \\
\hline 3 & 43765827 & 44445298 & parkinson protein 2, E3 ubiquitin protein ligase (parkin) & 0.750 \\
\hline 3 & 44773338 & 44826366 & cation-independent mannose-6-phosphate receptor precursor & 0.750 \\
\hline 3 & 44874173 & 44881975 & T-complex protein 1 subunit alpha & 0.750 \\
\hline 3 & 44881987 & 44889927 & acetyl-CoA acetyltransferase, cytosolic & 0.750 \\
\hline 3 & 44923214 & 44929994 & superoxide dismutase & 0.750 \\
\hline 3 & 45071689 & 45111718 & fibronectin type III domain containing 1 & 0.750 \\
\hline 3 & 45215648 & 45566922 & utrophin & 0.750 \\
\hline 3 & 45769623 & 45811385 & epilepsy, progressive myoclonus type $2 \mathrm{~A}$, Lafora disease (laforin) & 0.750 \\
\hline 3 & 45868769 & 45921436 & SNF2 histone linker PHD RING helicase, E3 ubiquitin protein ligase & 0.750 \\
\hline 3 & 45937157 & 46118743 & Glutamate receptor metabotropic 1 isoform $\mathrm{f}$ transcript variant 1 & 0.750 \\
\hline 3 & 46182574 & 46256838 & androglobin & 0.750 \\
\hline 3 & 46388675 & 46489008 & syntaxin binding protein 5 (tomosyn) & 0.750 \\
\hline 3 & 46522593 & 46647011 & sterile alpha motif domain containing 5 & 0.750 \\
\hline 3 & 47153562 & 47250156 & uronyl-2-sulfotransferase & 0.750 \\
\hline 3 & 47394890 & 47423136 & peptidylprolyl isomerase (cyclophilin)-like 4 & 0.750 \\
\hline 3 & 47425648 & 47442269 & uncharacterized protein 66 orf72 homolog precursor & 0.750 \\
\hline
\end{tabular}


3

$47446107 \quad 47459848$

4750353747537193 Protein-L-isoaspartate(D-aspartate) O-methy

3

3

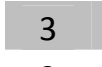

3

\begin{tabular}{|l|l|l|}
47538737 & 47606787 & low density lipoprotein receptor-related protein 11 \\
\hline 44874859 & 44874995 & Small nucleolar RNA SNORA29
\end{tabular}

4488014544880277 Small nucleolar RNA SNORA20

4827657148431441 estrogen receptor

4844703248720659 spectrin repeat containing, nuclear envelope 1

4877628448795822 VIP peptides isoform 2 preproprotein

4891015348981998 Regulator of G-protein signaling 17

5046576450754522 AT rich interactive domain 1B (SWI1-like)

$5324897753250435 \mathrm{cbp} / \mathrm{p} 300$-interacting transactivator 2

$5448537054505293 \quad \mathrm{BCL} 2$-associated transcription factor 1

5802986258422557 protein tyrosine phosphatase, receptor type, K

3

6322937763243518 RWD domain containing 1

6326126163269158 uncharacterized protein LOC769904

0.750

0.750

0.750

0.750

0.750

0.758

0.758

0.758

0.758

0.731

0.511

0.555

0.530

63269158 uncharacterized protein LOC769904 0.673

6327614363280172 family with sequence similarity 26, member F 0.673

6343164763439612 Collagen alpha-1(X) chain 0.673

6347238763519299 fyn-related kinase 0.673

6423938964261685 histone deacetylase $2 \quad 0.673$

36539200065429033 tyrosine-protein kinase Fyn 0.577

36554281165609587 REV3-like, polymerase (DNA directed), zeta, catalytic subunit 0.577

$365721376 \quad 65726791$ general transcription factor 3C polypeptide 6

36574404165761269 S-adenosylmethionine decarboxylase proenzyme 0.577

36603322966074352 pre-mRNA-processing factor 17

36620189666268774 polyphosphoinositide phosphatase precursor 0.577

36626957866325155 adenylate kinase 9

36775713167863760 Blimp-1 0.575

$3 \quad 7331896473462488$ Ephrin type-A receptor 7

37421044474210526 U6 spliceosomal RNA 0.667

37980279479856460 Interphotoreceptor matrix proteoglycan 1 


\begin{tabular}{|c|c|c|c|c|}
\hline 3 & 79860207 & 79930603 & myosin-VI & 0.675 \\
\hline 3 & 79966253 & 79995357 & SUMO1/sentrin specific peptidase 6 & 0.675 \\
\hline 3 & 80071280 & 80164378 & filamin A interacting protein 1 & 0.675 \\
\hline 3 & 80180051 & 80193545 & cell cycle control protein 50A & 0.675 \\
\hline 3 & 80197965 & 80201301 & cytochrome c oxidase subunit 7A2, mitochondrial & 0.675 \\
\hline 3 & 80212182 & 80312375 & collagen alpha-1(XII) chain precursor & 0.675 \\
\hline 3 & 80775681 & 80855396 & CD109 molecule & 0.675 \\
\hline 3 & 81337238 & 81638081 & regulating synaptic membrane exocytosis 1 & 0.578 \\
\hline 3 & 86044771 & 86130756 & DNA primase large subunit & 0.540 \\
\hline 3 & 96729661 & 96737554 & PQ loop repeat containing 3 & 0.721 \\
\hline 3 & 96746492 & 96802174 & Rho-associated, coiled-coil containing protein kinase 2 & 0.721 \\
\hline 3 & 96852537 & 96861622 & transcription factor E2F6 & 0.721 \\
\hline 3 & 96906122 & 96965158 & growth regulation by estrogen in breast cancer 1 & 0.721 \\
\hline 3 & 96971362 & 97044067 & Lipin 1; Uncharacterized protein & 0.721 \\
\hline 3 & 97427885 & 97448910 & tribbles homolog 2 & 0.721 \\
\hline 3 & 98343307 & 98504175 & neuroblastoma amplified sequence & 0.721 \\
\hline 3 & 98505120 & 98526381 & ATP-dependent RNA helicase DDX1 & 0.721 \\
\hline 3 & 98778414 & 98780948 & $\mathrm{~N}$-myc proto-oncogene protein & 0.721 \\
\hline 3 & 99739837 & 99814889 & Visinin-like protein 1 & 0.551 \\
\hline 3 & $1.04 \mathrm{E}+08$ & $1.05 E+08$ & putative Polycomb group protein ASXL2 & 0.536 \\
\hline 4 & 32207746 & 32286859 & doublecortin-like kinase 2 & 0.799 \\
\hline 4 & 46222167 & 46249662 & Phosphatidate cytidylyltransferase & 0.553 \\
\hline 4 & 49571259 & 49794271 & solute carrier family 4 , sodium bicarbonate cotransporter, member 4 & 0.699 \\
\hline 4 & 49817085 & 49822899 & vitamin D-binding protein precursor & 0.699 \\
\hline 4 & 49832925 & 49847032 & neuropeptide FF receptor 2 & 0.699 \\
\hline 4 & 49890856 & 49977356 & ADAM metallopeptidase with thrombospondin type 1 motif, 3 & 0.699 \\
\hline 4 & 50153707 & 50226185 & ankyrin repeat domain 17 & 0.699 \\
\hline 4 & 50253145 & 50269174 & serum albumin precursor & 0.699 \\
\hline 4 & 50342296 & 50345374 & interleukin-8 precursor & 0.699 \\
\hline 4 & 50355001 & 50359957 & Interleukin-8 & 0.699 \\
\hline
\end{tabular}




\begin{tabular}{|c|c|c|c|c|}
\hline 4 & 50470435 & 50669744 & ephrin type-A receptor 5 precursor & 0.699 \\
\hline 4 & 50510247 & 50510326 & Small nucleolar RNA R12 & 0.699 \\
\hline 4 & 55856553 & 55899875 & 2-hydroxyacylsphingosine 1-beta-galactosyltransferase precursor & 0.551 \\
\hline 4 & 56052213 & 56091725 & arylsulfatase family, member J & 0.551 \\
\hline 4 & 58208189 & 58447378 & netrin receptor UNC5C precursor & 0.534 \\
\hline 4 & 59910288 & 60049717 & protein phosphatase 3 , catalytic subunit, alpha isozyme & 0.616 \\
\hline 4 & 69781392 & 69796531 & death domain containing 1 & 0.537 \\
\hline 4 & 69799893 & 69923066 & ArfGAP with RhoGAP domain, ankyrin repeat and PH domain 2 & 0.537 \\
\hline 4 & 75401346 & 75452145 & Ligand-dependent nuclear receptor corepressor-like protein & 0.732 \\
\hline 4 & 75480300 & 75503309 & non-SMC condensin I complex, subunit G & 0.732 \\
\hline 4 & 81411667 & 81420730 & alpha-2-macroglobulin receptor-associated protein precursor & 0.584 \\
\hline 4 & 81465866 & 81491136 & docking protein 7 & 0.584 \\
\hline 4 & 81495986 & 81536120 & HGF activator & 0.584 \\
\hline 4 & 81642832 & 81659465 & Myb/SANT-like DNA-binding domain containing 1 & 0.584 \\
\hline 4 & 81699702 & 81774162 & huntingtin & 0.584 \\
\hline 4 & 81780294 & 81810730 & G protein-coupled receptor kinase 4 & 0.584 \\
\hline 4 & 81861191 & 81918087 & alpha-adducin & 0.584 \\
\hline 4 & 81924388 & 81959922 & SH3 domain-binding protein 2 & 0.584 \\
\hline 4 & 82006422 & 82013592 & TNFAIP3-interacting protein 2 & 0.584 \\
\hline 4 & 82049672 & 82097810 & family with sequence similarity 193, member A & 0.584 \\
\hline 4 & 82143274 & 82159327 & RING finger protein 4 & 0.584 \\
\hline 4 & 82408395 & 82444435 & max dimerization protein 4 & 0.584 \\
\hline 4 & 82459166 & 82544586 & polymerase (DNA directed) nu & 0.584 \\
\hline 4 & 81902213 & 81902370 & U1 spliceosomal RNA & 0.584 \\
\hline 5 & 10010798 & 10123603 & inscuteable homolog (Drosophila) & 0.626 \\
\hline 5 & 12238926 & 12267404 & receptor-type tyrosine-protein phosphatase eta & 0.755 \\
\hline 5 & 13148583 & 13163279 & Insulin-like growth factor II & 0.537 \\
\hline 5 & 13377709 & 13388547 & $39 S$ ribosomal protein $\mathrm{L} 23$, mitochondrial & 0.537 \\
\hline 5 & 13389730 & 13416288 & Troponin T, fast skeletal muscle isoforms & 0.537 \\
\hline 5 & 13434000 & 13472383 & Iymphocyte-specific protein 1 & 0.537 \\
\hline
\end{tabular}



rhombotin-2 
5

5

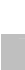

5

5
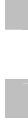

3863450038651966 SNW domain containing 1

3865738038732532 uncharacterized aarF domain-containing protein kinase 1 precursor

3982622939837879 lodothyronine deiodinase

4004254040090213 thyrotropin receptor isoform 1 precursor

$40099534 \quad 40119234$ transcription initiation factor IIA subunit 1

4014482840210981 stonin 2

4231973042344911 spermatogenesis associated 7

4806783148083159 cholesterol 24-hydroxylase

4815964848241305 echinoderm microtubule associated protein like 1

4831277148378821 ena/VASP-like protein 0.595

54841807748431424 transcriptional repressor protein YY1

4845682848465791 solute carrier family 25, member 47

5

5

5
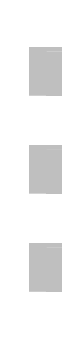

\begin{tabular}{|r|r|r|ll}
\hline 6 & 12928166 & 13365708 & calcium-activated potassium channel subunit alpha-1 & 0.546 \\
\hline 6 & 19439617 & 19465235 & tankyrase-2 & \\
\hline 6 & 20586643 & 20593197 & cyclin J & 0.564 \\
\hline 6 & 20638215 & 20730717 & B-cell linker protein & 0.614 \\
\hline 6 & 20754954 & 20843012 & DNA nucleotidylexotransferase & 0.614 \\
\hline 6 & 21051932 & 21053666 & VENT homeobox & 0.614 \\
\hline 6 & 21074071 & 21135637 & kinase non-catalytic C-lobe domain (KIND) containing 1 & 0.614 \\
\hline 7 & 3031382 & 3433912 & receptor tyrosine-protein kinase erbB-4 & 0.614 \\
\hline
\end{tabular}

0.595

0.573

0.573

0.634

0.634

0.634

0.612

0.595

0.595

0.595

0.603

0.603

0.622

0.622

0.519

0.628

0.628

0.628

546

14

614

0.614

0.517 


\begin{tabular}{|c|c|c|c|c|}
\hline 7 & 4722132 & 4740447 & melanophilin & 0.543 \\
\hline 7 & 16404276 & 16412287 & secernin 3 & 0.623 \\
\hline 7 & 16412437 & 16433377 & Corepressor interacting with RBPJ 1 & 0.623 \\
\hline 7 & 16463674 & 16548896 & Obg-like ATPase 1 & 0.623 \\
\hline 7 & 17199467 & 17208239 & pyruvate dehydrogenase kinase, isozyme 1 & 0.639 \\
\hline 7 & 17214612 & 17252865 & integrin alpha-6 precursor & 0.639 \\
\hline 7 & 17770145 & 17966292 & myosin IIIB & 0.547 \\
\hline 7 & 18583602 & 18668725 & serine threonine kinase 39 & 0.652 \\
\hline 7 & 21293508 & 21321944 & Integrin beta & 0.594 \\
\hline 7 & 21327981 & 21363749 & secretory phospholipase $\mathrm{A} 2$ receptor precursor & 0.594 \\
\hline 7 & 26451247 & 26646791 & adenylate cyclase type 5 & 0.624 \\
\hline 7 & 26653371 & 26673051 & protein-tyrosine phosphatase-like member B & 0.624 \\
\hline 7 & 26674970 & 26859021 & myosin light chain kinase, smooth muscle & 0.624 \\
\hline 7 & 30459507 & 30701723 & thrombospondin, type I, domain containing 7B & 0.539 \\
\hline 7 & 31350097 & 31932431 & low density lipoprotein receptor-related protein 1 & 0.549 \\
\hline 7 & 35234890 & 35264450 & G protein-activated inward rectifier potassium channel 1 & 0.525 \\
\hline 8 & 822750 & 960453 & netrin G1 & 0.799 \\
\hline 8 & 987129 & 1134223 & guanine nucleotide exchange factor VAV3 & 0.799 \\
\hline 8 & 2165461 & 2220563 & serine/threonine-protein kinase Nek7 & 0.532 \\
\hline 8 & 5890359 & 5957040 & acyl-CoA binding domain containing 6 & 0.637 \\
\hline 9 & 2069219 & 2087633 & kelch-like 6 & 0.531 \\
\hline 9 & 5411909 & 5436836 & retinol binding protein 1 , cellular & 0.731 \\
\hline 9 & 5566977 & 5861817 & calsyntenin 2 & 0.731 \\
\hline 9 & 7800341 & 7823434 & plasminogen activator inhibitor type 1 , member 2 precursor & 0.563 \\
\hline 9 & 12913816 & 12999648 & fibroblast growth factor 12 & 0.629 \\
\hline 9 & 13178354 & 13185135 & urotensin-2B precursor & 0.629 \\
\hline 9 & 13186472 & 13199826 & osteocrin precursor & 0.629 \\
\hline 9 & 20419761 & 20464371 & cholinesterase precursor & 0.605 \\
\hline 9 & 23218003 & 23220395 & profilin-2 & 0.780 \\
\hline 9 & 23226057 & 23254253 & E3 ubiquitin-protein ligase RNF13 & 0.780 \\
\hline
\end{tabular}


23264511

23304671

COMM domain containing 2$$
\text { (10) }
$$

23425241 glycogenin-1

0.780

103103512

3116708

0.511

103952464

(choline receptor subunit beta-4 precursor

0.511

$10 \quad 5072623$

$10 \quad 5146745$

$10 \quad 6434758$

$10 \quad 6480269$

3127878 Neuronal acetylcholine receptor subunit alpha-3

0.511

5098236 Kruppel-like factor 13

5227651 transient receptor potential cation channel, subfamily M, member 1

0.666

6619897 disintegrin and metalloproteinase domain-containing protein 10 precursor

0.671

64802696521283 lipase, hepatic

0.671

$10 \quad 6572777$

$10 \quad 6604818$

6596733 aquaporin 9

6816944

6661741

Retinal dehydrogenase 2

0.671

\begin{tabular}{l|l}
10 & 68169 \\
10 & 69032
\end{tabular}

6857906 cingulin-like 1

$10 \quad 19265373$

7058903

transcription

0.671

0.671

0.671

$10 \quad 19310281$

$11 \quad 43576$

19502893

0.595

19330984 protein CASC4 precursor

0.595

4419746 RPGRIP1-like

$4456387 \quad 4469147 \quad$ AKT-interacting protein

0.540

1110016612

10144234

0.540

$\begin{array}{lll}11 & 10168414 & 10216329\end{array}$

actosamine 4-0) sulfotransferase 8

0.644

$11 \quad 10356455$

10374076

$\begin{array}{lll}11 & 10376830 & 10403785\end{array}$

0.644

$11 \quad 10450875$

10471700

protein LS14 homolog A

0.644

0.644

$\begin{array}{lll}11 & 10610357 \quad 10619189\end{array}$

glucose-6-phosphate isomerase

0.644

0.644

\begin{tabular}{l|l|l|l}
11 & 10624075 & 1062909
\end{tabular}

$\begin{array}{lll}11 & 10773808 & 10782445\end{array}$

programmed cell death protein 2-like

0.644

$11 \quad 1078661010798981$ NEDD8-activating enzyme E1 regulatory subunit

0.644

$11 \quad 10817084$

10841597

NEDD8-activating enzyme E1 regulatory subunit

0.644

111089438610903372 CKLF-like MARVEL transmembrane domain-containing protein 3 


\begin{tabular}{|c|c|c|c|c|}
\hline 11 & 10906480 & 10917656 & thymidine kinase 2 , mitochondrial & 0.644 \\
\hline 11 & 10927995 & 10964663 & protein BEAN1 & 0.644 \\
\hline 11 & 10982217 & 11011468 & cadherin-5 precursor & 0.644 \\
\hline 11 & 11582884 & 11662192 & cadherin-11 precursor & 0.644 \\
\hline 11 & 10763464 & 10765059 & pyruvate dehyrogenase phosphatase catalytic subunit 2 & 0.644 \\
\hline 11 & 12288497 & 12432361 & cadherin-8 & 0.661 \\
\hline 11 & 12638991 & 12639143 & TUC338 & 0.661 \\
\hline 11 & 18267773 & 18286192 & transcription factor 25 (basic helix-loop-helix) & 0.667 \\
\hline 11 & 18290821 & 18292152 & tubulin beta- 4 chain & 0.667 \\
\hline 11 & 18293266 & 18296749 & differentially expressed in FDCP 8 homolog (mouse) & 0.667 \\
\hline 11 & 18300555 & 18308190 & growth arrest-specific 8 & 0.667 \\
\hline 11 & 18308197 & 18309804 & urate (hydroxyiso-) hydrolase, pseudogene & 0.667 \\
\hline 11 & 18310977 & 18316940 & cadherin 3, type 1, P-cadherin (placental) & 0.667 \\
\hline 11 & 18317572 & 18326276 & cadherin-1 precursor & 0.667 \\
\hline 11 & 18355336 & 18357463 & hyaluronan synthase 3 & 0.667 \\
\hline 11 & 18358802 & 18360418 & CTF8, chromosome transmission fidelity factor 8 homolog (S. cerevisiae) & 0.667 \\
\hline 11 & 18365413 & 18370296 & syntrophin, beta 2 (dystrophin-associated protein $A 1,59 \mathrm{kDa}$, basic component 2 ) & 0.667 \\
\hline 11 & 18378690 & 18380054 & 605 ribosome subunit biogenesis protein NIP7 homolog & 0.667 \\
\hline 11 & 18383995 & 18391771 & Telomeric repeat-binding factor 2 & 0.667 \\
\hline 11 & 18396032 & 18407915 & outer mitochondrial membrane cytochrome b5 & 0.667 \\
\hline 11 & 18415930 & 18474957 & nuclear factor of activated T-cells 5 & 0.667 \\
\hline 11 & 18482780 & 18484568 & $\mathrm{NAD}(\mathrm{P}) \mathrm{H}$ dehydrogenase, quinone 1 & 0.667 \\
\hline 11 & 18487655 & 18519315 & NEDD4-like E3 ubiquitin-protein ligase WWP2 & 0.667 \\
\hline 11 & 18520346 & 18526403 & proteasome (prosome, macropain) 26S subunit, non-ATPase, 7 & 0.667 \\
\hline 11 & 19150949 & 19161411 & pre-mRNA-splicing factor ATP-dependent RNA helicase PRP16 & 0.667 \\
\hline 11 & 18287877 & 18288821 & melanocyte-stimulating hormone receptor & 0.667 \\
\hline 12 & 13983652 & 14275283 & membrane associated guanylate kinase, WW and PDZ domain containing 1 & 0.559 \\
\hline 12 & 18847535 & 19073767 & glutamate receptor, metabotropic 7 & 0.581 \\
\hline 13 & 4187413 & 4738485 & teneurin-2 isoform 1 & 0.685 \\
\hline 13 & 10737034 & 10764716 & IL2-inducible T-cell kinase & 0.565 \\
\hline
\end{tabular}




\begin{tabular}{|c|c|c|c|c|}
\hline 13 & 13243833 & 13260270 & glutamine-fructose-6-phosphate transaminase 2 & 0.528 \\
\hline 13 & 13261655 & 13281786 & Mitogen-activated protein kinase 9 & 0.528 \\
\hline 13 & 16744797 & 16844189 & Rho GTPase-activating protein 26 & 0.567 \\
\hline 14 & 6602197 & 6621651 & leucine carboxyl methyltransferase 1 & 0.598 \\
\hline 15 & 2912110 & 3202073 & syntaxin-2 & 0.595 \\
\hline 15 & 3051055 & 3169587 & G protein-coupled receptor 133 & 0.595 \\
\hline 15 & 3179902 & 3184192 & GTP-binding nuclear protein Ran & 0.595 \\
\hline 15 & 3029980 & 3030156 & TUC338 & 0.595 \\
\hline 15 & 4855484 & 4908313 & dynein, axonemal, heavy chain 10 & 0.636 \\
\hline 15 & 4909039 & 4924155 & V-type proton ATPase $116 \mathrm{kDa}$ subunit a isoform 2 & 0.636 \\
\hline 15 & 4933001 & 4937788 & general transcription factor $\mathrm{IIH}$, polypeptide $3,34 \mathrm{kDa}$ & 0.636 \\
\hline 15 & 4941748 & 4948314 & ATP-dependent RNA helicase DDX55 & 0.636 \\
\hline 15 & 4977536 & 4979234 & small nuclear ribonucleoprotein 35kDa (U11/U12) & 0.636 \\
\hline 15 & 4986361 & 4990790 & SET domain containing (lysine methyltransferase) 8 & 0.636 \\
\hline 15 & 5002524 & 5026119 & protein strawberry notch homolog 1 & 0.636 \\
\hline 15 & 5038370 & 5051012 & cyclin-dependent kinase 2 -associated protein 1 & 0.636 \\
\hline 15 & 5053460 & 5077681 & M-phase phosphoprotein 9 & 0.636 \\
\hline 15 & 5080304 & 5188058 & phosphatidylinositol transfer protein, membrane-associated 2 & 0.636 \\
\hline 15 & 5192449 & 5195746 & ADP-ribosylation factor-like protein 6-interacting protein 4 & 0.636 \\
\hline 15 & 5208507 & 5219304 & ATP-binding cassette, sub-family B (MDR/TAP), member 9 & 0.636 \\
\hline 15 & 5243010 & 5257347 & huntingtin-interacting protein 1-related protein & 0.636 \\
\hline 15 & 5264075 & 5270199 & Density-regulated protein & 0.636 \\
\hline 15 & 5297891 & 5339287 & Sarcoplasmic/endoplasmic reticulum calcium ATPase 2 & 0.636 \\
\hline 15 & 6301498 & 6347392 & HECT domain containing E3 ubiquitin protein ligase 4 & 0.514 \\
\hline 18 & 297153 & 334809 & myosin, heavy chain 13, skeletal muscle & 0.662 \\
\hline 18 & 2569645 & 2666638 & protoheme IX farnesyltransferase, mitochondrial & 0.544 \\
\hline 18 & 4721036 & 4751867 & SAP30 binding protein & 0.662 \\
\hline 18 & 4750809 & 4787690 & RecQ protein-like 5 & 0.662 \\
\hline 18 & 4763901 & 4774954 & uncharacterized protein LOC770371 & 0.662 \\
\hline 18 & 6953318 & 6958961 & importin subunit alpha-2 & 0.645 \\
\hline
\end{tabular}




\begin{tabular}{|c|c|c|c|c|}
\hline 18 & 6970301 & 7017419 & bromodomain PHD finger transcription factor & 0.645 \\
\hline 18 & 7017750 & 7029054 & nucleolar protein 11 & 0.645 \\
\hline 18 & 7205116 & 7244436 & voltage-dependent calcium channel gamma- 4 subunit & 0.645 \\
\hline 18 & 7895218 & 7917904 & axin-2 & 0.598 \\
\hline 18 & 7997936 & 8029708 & regulator of G-protein signaling 9 & 0.598 \\
\hline 18 & 8091453 & 8108190 & arylsulfatase $\mathrm{G}$ & 0.598 \\
\hline 18 & 8136004 & 8149977 & CAMP-dependent protein kinase type I-alpha regulatory subunit & 0.598 \\
\hline 18 & 8153051 & 8162773 & family with sequence similarity 20 , member $A$ & 0.598 \\
\hline 18 & 8232456 & 8254284 & ATP-binding cassette, sub-family A (ABC1), member 5 & 0.598 \\
\hline 18 & 8260526 & 8295962 & mitogen-activated protein kinase kinase 6 & 0.598 \\
\hline 18 & 8467064 & 8473808 & inward rectifier potassium channel 2 & 0.598 \\
\hline 19 & 7382617 & 7394940 & ribosomal protein 56 kinase beta- 1 & 0.575 \\
\hline 20 & 593577 & 597282 & TBC/LysM-associated domain containing 2 & 0.667 \\
\hline 20 & 597387 & 623826 & SAM domain and HD domain-containing protein 1 & 0.667 \\
\hline 20 & 626685 & 647924 & retinoblastoma-like 1 (p107) & 0.667 \\
\hline 20 & 650631 & 698519 & chromodomain helicase DNA binding protein 6 & 0.667 \\
\hline 20 & 731978 & 793938 & ral GTPase-activating protein subunit beta & 0.667 \\
\hline 20 & 835504 & 867279 & NDRG family member 3 & 0.667 \\
\hline 20 & 869594 & 872258 & Src-like-adaptor 2 & 0.667 \\
\hline 20 & 875509 & 927530 & PHD finger protein 20 & 0.667 \\
\hline 20 & 1049215 & 1303743 & copine I & 0.667 \\
\hline 20 & 1135479 & 1137453 & reactive oxygen species modulator 1 & 0.667 \\
\hline 20 & 1137517 & 1148952 & cysteine desulfurase, mitochondrial & 0.667 \\
\hline 20 & 1438852 & 1442860 & growth/differentiation factor 5 precursor & 0.667 \\
\hline 20 & 1510312 & 1559445 & ubiquinol-cytochrome $\mathrm{c}$ reductase complex chaperone & 0.667 \\
\hline 20 & 1565847 & 1590302 & family with sequence similarity 83, member $C$ & 0.667 \\
\hline 20 & 1591146 & 1598063 & eukaryotic translation initiation factor 6 & 0.667 \\
\hline 20 & 1601528 & 1644296 & matrix metallopeptidase 24 (membrane-inserted) & 0.667 \\
\hline 20 & 1643850 & 1862123 & adenosylhomocysteinase & 0.667 \\
\hline 20 & 1667567 & 1673056 & dynein, light chain, roadblock-type 1 & 0.667 \\
\hline
\end{tabular}




\begin{tabular}{|c|c|c|c|c|}
\hline 20 & 5699881 & 5712513 & adenosine deaminase & 0.757 \\
\hline 20 & 5740430 & 5750933 & serine incorporator 3 precursor & 0.757 \\
\hline 20 & 5748294 & 5755903 & alpha-tocopherol transfer protein-like & 0.757 \\
\hline 20 & 5763173 & 5777781 & hepatocyte nuclear factor 4-alpha & 0.757 \\
\hline 20 & 5792281 & 5795875 & R3H domain containing-like & 0.757 \\
\hline 20 & 5837979 & 5845069 & uncharacterized protein C20orf111 homolog & 0.757 \\
\hline 20 & 5847927 & 5855091 & junctophilin 2 & 0.757 \\
\hline 20 & 5895586 & 5918161 & solute carrier family 13 (sodium-dependent dicarboxylate transporter), member 3 & 0.757 \\
\hline 20 & 5923593 & 5925045 & TP53 regulating kinase & 0.757 \\
\hline 20 & 5928136 & 5935198 & solute carrier family 2 (facilitated glucose transporter), member 10 & 0.757 \\
\hline 20 & 6012484 & 6104467 & eyes absent homolog 2 & 0.757 \\
\hline 20 & 6203701 & 6257441 & nuclear receptor coactivator 3 & 0.757 \\
\hline 20 & 6529946 & 6608286 & phosphatidylinositol-3,4,5-trisphosphate-dependent Rac exchange factor 1 & 0.757 \\
\hline 20 & 6685428 & 6743411 & ADP-ribosylation factor guanine nucleotide-exchange factor 2 (brefeldin A-inhibited) & 0.757 \\
\hline 20 & 6747113 & 6768662 & CSE1 chromosome segregation 1-like (yeast) & 0.757 \\
\hline 20 & 6780571 & 6803177 & double-stranded RNA-binding protein Staufen homolog 1 & 0.757 \\
\hline 20 & 6994464 & 7004637 & transglutaminase 3 & 0.757 \\
\hline 20 & 7082081 & 7177518 & phosphatase and actin regulator 3 & 0.757 \\
\hline 20 & 7219136 & 7226342 & family with sequence similarity 217 , member B & 0.757 \\
\hline 20 & 7702268 & 8001028 & cadherin-4 precursor & 0.757 \\
\hline 20 & 7216161 & 7216973 & protein phosphatase 1 , regulatory subunit 3D & 0.757 \\
\hline 20 & 6814040 & 6814131 & Small nucleolar SNORD12/SNORD106 & 0.757 \\
\hline 20 & 6811970 & 6812061 & Small nucleolar SNORD12/SNORD106 & 0.757 \\
\hline 20 & 6813048 & 6813139 & Small nucleolar SNORD12/SNORD106 & 0.757 \\
\hline 20 & 6814693 & 6814787 & Small nucleolar SNORD12/SNORD106 & 0.757 \\
\hline 20 & 8527860 & 8554223 & solute carrier organic anion transporter family member $4 \mathrm{~A} 1$ & 0.527 \\
\hline 20 & 8575357 & 8620192 & neurotensin receptor type 1 & 0.527 \\
\hline 20 & 8689083 & 8720874 & collagen alpha-3(IX) chain precursor & 0.527 \\
\hline 20 & 8779439 & 8785907 & Glucose-induced degradation protein 8 homolog & 0.527 \\
\hline 20 & 8837210 & 8837872 & class E basic helix-loop-helix protein 23 & 0.527 \\
\hline
\end{tabular}




\begin{tabular}{|c|c|c|c|c|}
\hline 20 & 10874294 & 10878191 & Iysosomal protective protein precursor & 0.510 \\
\hline 20 & 10878671 & 10881926 & phospholipid transfer protein precursor & 0.510 \\
\hline 20 & 10887208 & 10895373 & PDX1 C-terminal inhibiting factor 1 & 0.510 \\
\hline 21 & 3033463 & 3151837 & arginine-glutamic acid dipeptide (RE) repeats & 0.517 \\
\hline 21 & 4679337 & 4732155 & F-actin-capping protein subunit beta isoforms 1 and 2 & 0.634 \\
\hline 21 & 4764694 & 4774114 & Neuroblastoma suppressor of tumorigenicity 1 & 0.634 \\
\hline 21 & 4776632 & 4780155 & 5-hydroxytryptamine receptor 6 & 0.634 \\
\hline 21 & 4822545 & 4823109 & ring finger protein 186 & 0.634 \\
\hline 21 & 4825556 & 4833537 & OTU domain containing 3 & 0.634 \\
\hline 21 & 4835306 & 4836901 & group IIE secretory phospholipase A2 precursor & 0.634 \\
\hline 21 & 4849821 & 4857034 & ATP-dependent RNA helicase DDX19B & 0.634 \\
\hline 21 & 4890645 & 4891820 & family with sequence similarity 43 , member B & 0.634 \\
\hline 21 & 4893462 & 4897243 & cytidine deaminase & 0.634 \\
\hline 21 & 4897971 & 4906221 & PTEN induced putative kinase 1 & 0.634 \\
\hline 21 & 4906433 & 4908768 & Agmatinase, mitochondrial & 0.634 \\
\hline 21 & 4909535 & 4918231 & dnaJ homolog subfamily $\mathrm{C}$ member 16 precursor & 0.634 \\
\hline 21 & 4918316 & 4924583 & caspase 9 , apoptosis-related cysteine peptidase & 0.634 \\
\hline 21 & 4925271 & 4927174 & chymotrypsin C (caldecrin) & 0.634 \\
\hline 21 & 4931027 & 4938907 & forkhead-associated (FHA) phosphopeptide binding domain 1 & 0.634 \\
\hline 21 & 4965242 & 4973503 & kazrin, periplakin interacting protein & 0.634 \\
\hline 21 & 5606164 & 5615523 & methylenetetrahydrofolate reductase $(\mathrm{NAD}(\mathrm{P}) \mathrm{H})$ & 0.571 \\
\hline 23 & 203721 & 213621 & UBX domain protein 11 & 0.560 \\
\hline 23 & 230940 & 237139 & connector enhancer of kinase suppressor of Ras 1 & 0.560 \\
\hline 23 & 861071 & 915914 & human immunodeficiency virus type I enhancer binding protein 3 & 0.597 \\
\hline 24 & 1073171 & 1109460 & GIRK4; Uncharacterized protein & 0.554 \\
\hline 24 & 6163150 & 6165998 & interleukin-18 & 0.543 \\
\hline 24 & 6167375 & 6171382 & succinate dehydrogenase & 0.543 \\
\hline 24 & 6176797 & 6179439 & PIH1 domain containing 2 & 0.543 \\
\hline 25 & 1156086 & 1174496 & coatomer subunit alpha & 0.524 \\
\hline 25 & 1174614 & 1186654 & nicastrin precursor & 0.524 \\
\hline
\end{tabular}




\begin{tabular}{|c|c|c|c|c|}
\hline 26 & 3399930 & 3416570 & CTTNBP2 N-terminal like & 0.518 \\
\hline 26 & 3436365 & 3447136 & protein Wnt-2b & 0.518 \\
\hline 26 & 3455410 & 3474542 & Suppressor of tumorigenicity 7 protein-like & 0.518 \\
\hline 26 & 3474674 & 3483306 & F-actin-capping protein subunit alpha-1 & 0.518 \\
\hline 26 & 3486369 & 3492328 & putative helicase MOV-10 & 0.518 \\
\hline 26 & 3492792 & 3497763 & Rho-related GTP-binding protein RhoC & 0.518 \\
\hline 26 & 3499167 & 3506175 & protein phosphatase, $\mathrm{Mg} 2+/ \mathrm{Mn} 2+$ dependent, $1 \mathrm{~J}$ & 0.518 \\
\hline 26 & 3552668 & 3567609 & monocarboxylate transporter 1 & 0.518 \\
\hline 26 & 3631292 & 3656668 & membrane-associated guanylate kinase, WW and PDZ domain-containing protein 3 & 0.518 \\
\hline Z & 22034805 & 22073103 & PAP associated domain containing 4 & 0.802 \\
\hline Z & 25582286 & 25666831 & dedicator of cytokinesis 8 & 1.000 \\
\hline Z & 25808415 & 25864441 & doublesex- and mab-3-related transcription factor 1 & 1.000 \\
\hline Z & 25902813 & 25905855 & doublesex and mab-3 related transcription factor 2 & 1.000 \\
\hline Z & 26195620 & 26294557 & SWI/SNF related, matrix associated, actin dependent regulator of chromatin, subfamily a, member 2 & 1.000 \\
\hline Z & 26415507 & 26430542 & very low density lipoprotein receptor & 1.000 \\
\hline Z & 26488495 & 26512565 & KIAA0020 & 1.000 \\
\hline Z & 26531633 & 26586078 & regulatory factor X, 3 (influences HLA class II expression) & 1.000 \\
\hline Z & 26708083 & 26866140 & GLIS family zinc finger 3 & 1.000 \\
\hline Z & 35753341 & 35806349 & retinal dehydrogenase 1 & 0.858 \\
\hline Z & 35881518 & 35899231 & annexin $\mathrm{A} 1$ & 0.858 \\
\hline Z & 39039875 & 39114050 & transducin-like enhancer of split 1 (E(sp1) homolog, Drosophila) & 0.952 \\
\hline Z & 39574349 & 39615592 & RAS and EF-hand domain containing & 0.952 \\
\hline Z & 39667818 & 39812156 & FERM domain containing 3 & 0.952 \\
\hline Z & 39900490 & 39924774 & kinesin family member 27 & 0.952 \\
\hline Z & 39936510 & 39954264 & Heterogeneous nuclear ribonucleoprotein $\mathrm{K}$ & 0.952 \\
\hline Z & 39954655 & 39958510 & recQ-mediated genome instability protein 1 & 0.952 \\
\hline Z & 38576413 & 38576570 & U1 spliceosomal RNA & 0.952 \\
\hline Z & 42249104 & 42257660 & uncharacterized protein LOC427470 & 1.000 \\
\hline Z & 42279475 & 42284756 & Cryptic protein & 1.000 \\
\hline Z & 42326478 & 42396311 & leucine rich repeat containing 2 & 1.000 \\
\hline
\end{tabular}


pindlin-Z

1.000

4331188843377895 SHC (Src homology 2 domain containing) transforming protein 3$$
\text { Z }
$$ 
6234474562440821 Hyaluronan and proteoglycan link protein 1

6357495063597283 creatine kinase S-type, mitochondrial

6360195363729018 Ras protein-specific guanine nucleotide-releasing factor 2

Z 6375985563876936 mutS homolog 3 (E. coli)

Z 6387664563893410 dihydrofolate reductase

6398474363988783 adenylate kinase isoenzyme 6

0.926

Z 6398863164006407 cell cycle checkpoint protein RAD17

0.926

Z 6564435565663759 A-kinase anchor protein 2

Z $65673926 \quad 65679018$ solute carrier family 46, member 2

0.869

Z 6568556865734943 sorting nexin family member 30

Z 6574496565755449 SOSS complex subunit C 